\title{
The ODESeW Platform as a Tool for Managing EU Projects: The Knowledge Web Case Study
}

\author{
Asunción Gómez-Pérez ${ }^{1}$, Angel López-Cima ${ }^{1}$, \\ M. Carmen Suárez-Figueroa ${ }^{1}$, and Oscar Corcho ${ }^{2}$ \\ ${ }^{1}$ OEG - Facultad de Informática. Universidad Politécnica de Madrid (UPM) Campus de \\ Montegancedo, s/n. 28660 Boadilla del Monte. Madrid. Spain \\ \{asun, alopez, mcsuarez\}@fi.upm.es \\ ${ }^{2}$ University of Manchester. School of Computer Science. Oxford Road, Manchester, United \\ Kingdom \\ Oscar.Corcho@manchester.ac.uk
}

\begin{abstract}
ODESeW allows developing ontology-based Web portals. It provides functionalities to edit and browse information, taking into account access privileges, and to update automatically changes carried out on the underlying ontologies. In this paper, we describe the ontologies used in a specific deployment of the ODESeW platform, oriented to the management of EU R\&D projects. We also present the functions offered in this specific deployment, giving as an example the EU Knowledge Web Network of Excellence portal.
\end{abstract}

\section{Introduction}

One important aspect of project management is the generation of reports on the current state of the project, so that the project progress can be monitored by project members or by outsiders. The quality of the project documentation thus generated has an important influence on the level of detail of the monitoring that can be performed, on decision-making, and on other project management activities.

Let us focus on the specific setting of the R\&D projects funded by the European Commission (EC). These projects are run by a consortium of several academic and industrial partners from different EU countries. Progress reports are not only issued to monitor the project evolution inside the consortium, but also to inform the EC project officer of that evolution. Project reporting requires input from every partner in the consortium. These inputs are submitted to the project coordinator, who is responsible for the generation and submission of the consolidated information. Normally, the project coordinator has to put in a huge effort to harvest these partial reports from each partner, to generate the consolidated documents and to maintain consistency among different versions of the partial reports, the project description and the original plan. This task is even harder if we consider that there are no specialized tools to help building reports, tracking changes and ensuring consistency.

In this paper we show how we have improved the EU R\&D project management by using an ontology-based project portal that provides, among other functionalities, a set of project management functions. These functions are based on knowledge of 
project management and reporting that has been formalized by a set of ontologies, and that has been used for the construction of several EU project portals. For our descriptions we will focus on the FP6 Network of Excellence (NoE) Knowledge Web ${ }^{1}$, which is the one posing more challenges and constraints given the amount of institutions, workpackages and deliverables.

To build such a project portal we have used the Semantic Web application development framework ODESeW [4]. This framework can be easily extended with new knowledge (new ontologies about the project management); besides, it eases the development of new advanced functionalities on top of the portal, since the semantics of the portal content is exposed explicitly. Finally, such a framework facilitates the reusability of knowledge and functionalities among applications, as explained in [4].

The paper is organized as follows: section 2 provides a brief description of the ODESeW framework; section 3 describes how periodic progress reports are structured in EU projects so that we can get a better idea of the amount of work involved in their generation; section 4 presents the ontologies used within the project portal. Section 5 gives a brief description of the functionalities provided by the portal. Finally, section 6 provides a conclusion and outlines a vision of the work to be done in the future.

\section{The Development Framework ODESeW}

ODESeW ( Semantic Web Portal based on WebODE) was first described in [2] as a tool that could be used for the automatic generation of Web portals in which all the information was indexed by means of ontologies. This system was built on top of the WebODE ontology engineering workbench [1], thus inheriting many of its features.

The portals generated with ODESeW could be used as the Intranets and Extranets of the projects, taking into account that different users will have different read and write permissions in the portal. Besides, ODESeW provided functions such as a search engine, content implementation in different languages (RDF, RDFS and OWL) and administrator functionalities for user management, read/write permission management, and a selection of ontologies to be used in the portal.

In this version of ODESeW, it switches from being a tool for building ontologybased portals into a more complete framework for building Semantic Web applications. This new version offers developers a set of services and tools that can be used in a Semantic Web application and gives, by default, navigation and visualization models that allow visualizing, editing and navigating the content in the portal. Such models can be modified and extended easily, permitting developers to create specific visualization and navigation models. The technical details are provided in [4].

\section{Project Management and Periodic Progress Reports}

As commented in the introduction, collaborative projects usually require the creation of periodic project progress reports that project coordinators can use to monitor the project and that, in the case of the EU R\&D projects, must be sent to the European

\footnotetext{
${ }^{1}$ http://knowledgeweb.semanticweb.org/
} 
Commission (EC) at regular intervals during the project execution. EC reports can be divided in the following sections:

1. Activity Report

1.1. Report on Workpackage (WP) activities

1.2. Published executive summary

1.3. Update plan for using and disseminating knowledge

1.4. Information about: publications, invited talks, workshop and conference organized, etc

2. Management Report

2.1. Financial statement

2.2. Summary financial report consolidating the costs of contractors

2.3. Audits certificates

2.4. Brief description of the work performed by each contractor during the period

2.5. Budgeted cost and actual costs

2.6. Budgeted person-month and actual person-month

2.7. Summary explanation of the impact of major deviations from cost budget and from person-month budget.

3. Distribution of EC funding to partners.

4. Interim Reports

The task of preparing a progress report normally consists of the following steps (which are executed by the project coordinator):

- Request progress reports from each of the partners involved in the consortium.

- Receive the partial progress reports and compile all that information in a single document, removing duplicates, detecting inconsistencies, etc. This step is performed iteratively until all the information is provided.

- Prepare a final summary, stressing the most important aspects of the results.

To help in these tasks, collaborative editing tools are used. However, much manual work must still be done, mainly on the project coordinator side, to make a good quality document for the EC. That is, a document containing all the information that is relevant inside the report period, with no duplicates, etc. It is important that the information there included be presented in a good homogeneous format.

\section{A Set of Ontologies for EU R\&D Project Management}

In this section we describe the domain model used in the Semantic Web application that we have created for EU R\&D project management. This domain model consists of a set of interlinked ontologies, namely, several project description ontologies, a user role ontology and a management ontology. We will give some details about the process followed to build such ontologies.

\subsection{The Ontology Building Process}

These ontologies have been developed following the METHONTOLOGY methodology [3]. One of the first tasks proposed by this methodology is that of searching for other ontologies in the same or in similar domains in order to reuse them (or part of them), so that we can avoid developing them form scratch. 
For developing the project description ontologies, we used Oyster ${ }^{2}$ [5] which permits searching for ontologies in similar domains. After analyzing the results provided by Oyster, the most appropriate ontologies were: the ontology used in the OntoWeb project $^{3}$ (Semantic Web Research Community Ontology ${ }^{4}$ ) and the ontologies used in the Esperonto project.

Once we identified which parts of the ontologies could be reused, we extended them following the steps proposed by the conceptualization phase of METHONTOLOGY. Thus, to extend the reused ontologies, we acquired the domain knowledge from the Knowledge Web Technical Annex. Besides, comments from project partners were considered in the refinement of these ontologies.

For the development of the user role and management ontologies, we did not reuse any ontology, we built them from scratch following the same methodology.

\subsection{Project Description Ontologies}

To describe a collaborative project we have used five ontologies, which can be easily reused for describing other similar projects. These ontologies are the following :

- The Documentation Ontology, which models knowledge of documentation used in the project. The main concept of this ontology is Documentation, a concept organized according to the type of document within a taxonomy.

- The Event Ontology, which models knowledge of events that are related to the project. The main concept of this ontology is Event.

- The Organization Ontology, which models knowledge of organizations involved in the project. The main concept in this ontology is Organization, a concept split into three subclasses. The most important information about organizations involved in a project is related to the organization itself and its location.

- The Person Ontology, which models knowledge of persons who work in the project. This ontology is focused on general-purpose personal information. The main concept of this ontology is Person. We have divided this concept into four different types: university staff, company staff, project officer, and student.

- The Project Ontology, which models the Technical Annex of a project, including information about WPs, tasks, projects, areas, etc. This ontology is not organized in a taxonomy; it only includes several concepts and the relationships between them.

\subsection{The User Role Ontology}

This ontology models the knowledge needed for managing different user profiles within the project. In the case of Knowledge Web, the roles that each user plays in the project are represented by each concept of the ontology.

ODESeW manages the user role ontology internally and independently of the application domain; this, however, does not mean that it cannot be extended for specific domains. Figure 1 shows the user roles that participate in the generation of progress reports and the extension needed for managing a NoE like Knowledge Web.

\footnotetext{
${ }^{2} \mathrm{http} / / /$ oyster.ontoware.org/

${ }^{3}$ http://www.ontoweb.org/

${ }^{4}$ http://ontobroker.semanticweb.org/ontos/swrc.html
} 


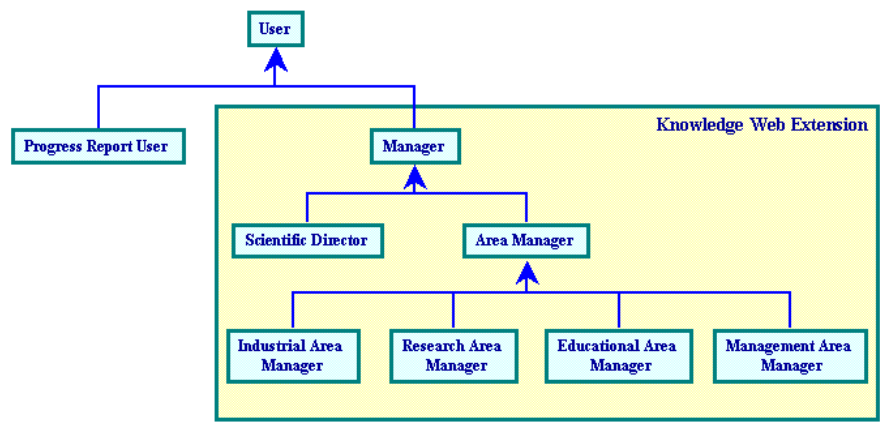

Fig. 1. The taxonomy of the user role ontology

\subsection{The Management Ontology}

This ontology contains the knowledge needed to manage collaborative projects. It identifies the most relevant concepts and properties used to collect information from partners and to generate the consolidated report. We can identify three main concepts:

- Period, which specifies different types of periods such as a Reporting Period or a Joint Process Activity (JPA) Period, that is, different periods identified in a project where the description of the work can be reviewed and changed.

- Effort, which represents the effort expended or to be expended in a project period, either in a task or a WP or by organization.

- Report, which represents a partial or complete report generated by a partner or by several partners. Partial reports are used to compile a complete report, which is then sent to the coordinator partner.

In the case of Knowledge Web, this ontology has been extended (as shown in Figure 2) with other partial reports, such as the Project Overview Report and different Area Reports, all required to create the complete report.

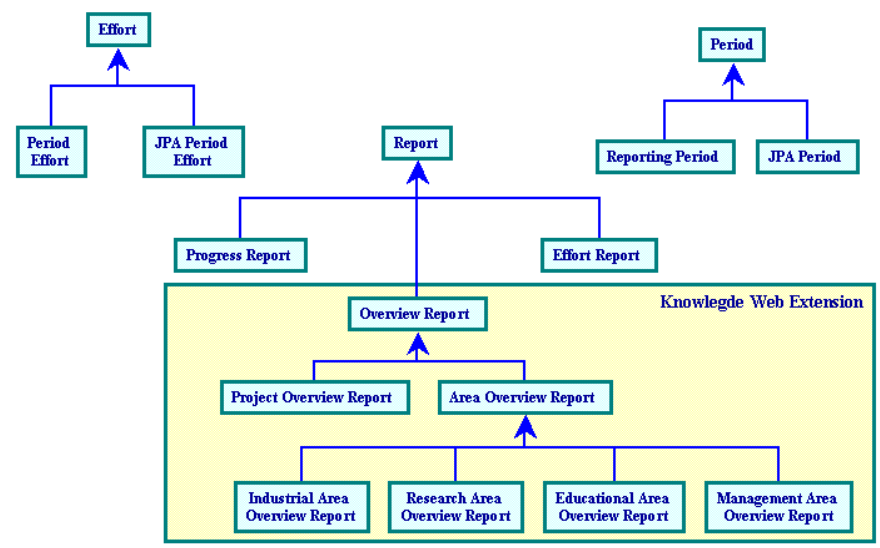

Fig. 2. The taxonomies of the management ontology 


\subsubsection{Periods}

The Period concept modeled in the management ontology represents different kinds of time frames during the project. The main periods are:

- The JPA Period, the period in which a project is divided. In each of these periods, the consortium defines the milestones per workpackage, the deliverables to report to the EC and the distribution of effort among the partners.

- The Reporting Period, which delimitates the time frame where the consortium must report progress inside a JPA Period. Depending on the project, this period could be every two or six months or every year.

\subsubsection{Efforts}

Two kinds of efforts can be found in the management ontology:

- JPA Period Effort, which represents the effort devoted by the consortium in each JPA Period on each WP.

- Periodic Effort, which is the effort expended by each partner on a specific WP in a Reporting Period.

\subsubsection{Report}

The management reports generated on each Periodic Report and supported by the current version for the reporting period are:

- The Effort Report, which represents the total effort put in during a Reporting Period by the whole consortium.

- The Progress Report, which represents the progress in a WP during a Reporting Period. In most of the projects, the data required in a progress report are: an overview, a description of the work carried out and the current status of each deliverable, the delays according to the definition of the project on each JPA, and the meetings held during the active period. Only the WP leader can include information in this report.

- The Area reports, which present an overview of all the work carried out during a Reporting Period in an Area. In Knowledge Web there are four areas (Industrial, Research, Educational and Management) and each of them is supported by a set of WPs. Each area has two area managers and only these persons can include information in the overview of their managed areas.

\section{Progress Reporting Functions}

Progress reporting functions are classified according to the user types. In the case of Knowledge Web there are 3 different user types: the reporting user, the area manager and the managing director. For the reporting user, the functions provided are oriented to guide the user through the different reports; for the area manager, the functions are oriented to generate the area progress reports; and, for the managing director, the functions are oriented to monitor the evolution of all the reports, to generate the complete reports and to produce other management reports. 


\subsection{Functions for Reporting Users}

When a reporting user logs into the system and enters the reporting section, the portal shows all the tasks to be done. These tasks are: WP progress reports, for each WP that the user's organization is leader of; and Effort report for the organization to which the user belongs.

The decision on which WPs and which effort reports are to be included is made on the basis of the user profile, which is obtained automatically by the portal. The portal also shows a time line with the schedules of the period reporting: WP and effort report stage, quality assessment, report updating, quality assurance and submission.

\subsection{Functions for Area Managers}

In a large project, WPs can be organized in different areas. Each area has a person that is responsible for it, known as the area leader. In the activity report, area managers can include an overview of the general progress.

\subsection{Functions for the Managing Director}

The managing director belongs to the project coordinator organization and is in charge of monitoring the progress of all reports produced by individual partners.

When a user logs into the system and accesses the reporting system, the portal shows the effort reports of all the project partners together with the progress reports of all the WPs. Besides, there is a link to a view for monitoring the current status of all reports, which shows a table that relates partners and WPs and also displays the current status of each progress and effort reported by the partner, using color coding.

The activity report is one of the documents that must be delivered by the project coordinator to the European Commission. This document compiles all the WP progress reports into one document. The document produced is presented in HTML and MS Word formats and it includes the front page, the table of contents, the header and footer of each page, and the font style selected by the project template. This document is a draft version which the managing director can modify with specific information that only the project coordinator is allowed to include.

\section{Conclusion and Future Work}

The current version of the EU R\&D project management portal covers section 1.1, some points of 1.4 and the section 2.6 of the management reports of an EC project (see Section 3). Such sections are the most collaboration-intensive parts of the generation process and the information to be used there is public to the consortium.

The use of these project management functions have reduced drastically the effort applied to producing management documentation for the EU R\&D project. These reports required collaboration from different partners in the project, which made the process time and effort consuming. This system reduces the amount of e-mails sent by the different project partners to the project coordinator, including the partial documents that have to be consolidated in a common version. It also reduces the number 
of errors that result from using intermediate versions of the documents and ensures that the results reported are consistent with the project description.

This tool also helps the project coordinator, represented by the managing director, to monitor the status of the current period report, detecting in this way any delay or major deviation with respect to the original workplan.

But the most important issue is that the progress report can be easily personalized for different projects just by changing or updating the existing ontologies, that is, including more fields in the report and different types of users in the report. The proof that this system works and can be reused is that we have used it (with the corresponding adaptations) in the context of several EU R\&D projects.

In the short term we plan to give full support for the generation of activity reports. We also plan to include more management documents, such as financial reports. Besides, we plan to support no only HTML and MS Word formats, as it has been done till now, but also other commonly used formats like LaTeX or the XML format that is being proposed by the EC as a common format for these reports.

\section{Acknowledgements}

This paper has been supported by the EU IST NoE Knowledge Web.

\section{References}

1. Arpírez, JC.; Corcho, O.; Fernández-López, M.; Gómez-Pérez, A. WebODE in a nutshell. AI Magazine 24(3):37-48. Fall 2003

2. Corcho, O.; Gómez-Pérez, A.; López-Cima, A.; López-García, V.; Suárez-Figueroa, M.C. 2003. "ODESeW. Automatic Generation of Knowledge Portals for Intranets and Extranets". International Semantic Web Conference 2003 (ISWC03). Lecture Notes in Computer Science (2870). PP: 802-817.

3. Fernández-López, M.; Gómez-Pérez, A.; Pazos-Sierra, A.; Pazos-Sierra, J. "Building a Chemical Ontology Using METHONTOLOGY and the Ontology Design Environment". IEEE Intelligent Systems \& their applications. January/February 1999. PP: 37-46.

4. López-Cima A.; Corcho O.; Gómez-Pérez A. 2006. A platform for the development of Semantic Web portals. In: Proceedings of the $6^{\text {th }}$ International Conference on Web Engineering (ICWE2006). Stanford, July 2006.

5. Palma, R.; Haase, P. 2005. "Oyster - Sharing and Re-using Ontologies in a Peer-to-Peer Community". International Semantic Web Conference 2005: 1059-1062. 